respectively. The station site is $550 \mathrm{ft}$. above sea. level, and both the sound and vision signals are radiated from the same aerial system, comprising two tiers of four vertical half-wave folded dipoles, erected at the top of a mast $750 \mathrm{ft}$. high. The design and method of feeding this aerial system from the transmitter are such as to give a horizontal radiationpattern, which is substantially uniform in all directions and over the required band of frequencies. A field-strength survey from the new station has been carried out by the B.B.C., and this has shown that, up to a range of about fifty miles, the field strength is generally greater than $0.5 \mathrm{mV} . / \mathrm{m}$. Based on experience of viewing conditions, it is considered that reception within this area will be good, but may be subject to interference where local conditions are severe. A useful signal of $0 \cdot 1 \mathrm{mV}$. $/ \mathrm{m}$. or more can be received at distances up to sixty-five miles from the transmitting station, but this may bo subject to severe interference and to some fading. Viewing conditions at greater distances, while still possible, will depend markodly on the terrain between trans. mitter and receiver and on the occurrence of favoura.ble weather conditions.

'The paper by Bevan and Page includes a description of the layout of the station and equipment at Sutton Coldfield, the power supplies and control mechanism, and the aerial and transmission-line system. The vision transmitter itself was described in more detail in the second paper, by E. A. Nind and E. McP. Leyton, in which it is pointed out that in some respects the new transmitter resembles that of the London station erected in 1936. In both cases high-level modulation is used; but in order to reduce liability to amplitude distortion, the output radio-frequency amplifier of the Sutton Coldfield. transmitter is cathode-driven, whereas in the former case grid-drive was used. The description of the transmitter given in this paper is general where the technique is well established, but is more detailed where novel parts of the equipment are concerned.

The third paper presented at the meeting, that by E. C. Cork, describes the design, construction and performance of the vestigial sideband. filter circuit installed at the Sutton Coldfield station. The basic design formulæ relevant to the filter network are given, together with a description of the practical circuit and construction adopted. The results of measurements of the attenuation characteristics of the filter show that there is good agreement between its performance and that of the idealized network.

These three papers form a very good and useful record of the design, construction and equipment of the first high-power television broadcasting station to be built in the world.

\section{INSTABILITY OF SMALL PLANETARY CORES}

$T$ WO papers, by W. H. Ramsey and M. J. Light. hill, respectively, "On the Instability of Small Planetary Cores", have recently been published (Mon. Not. Roy. Astro. Soc., 110, $4 ; 1950$ ). Previous papers by Ramsey on the subject, to which reference has been made in Nature ${ }^{1}$, suggested that the large jump in density at the boundary of the earth's core is not due to the appearance of new material but to a phase transition under pressure. Variation of density within the earth is, then, primarily due to the influence of pressure, and seismology provides the pressure-density relationship for the material. On the assumption that the other terrestrial planets have the same chemical composition as the earth, their mean densities can be computed, and Ramsey's results have been published ${ }^{2}$. In the course of the calculations it was found that very small cores are unstable and for this reason would not occur in Nature-a direct consequence of the main assumption that the jump in density at the boundary of the core is controlled by pressure.

A more comprehensive treatment of the subject is given in the two latest papers; in the first one, by Ramsey, it is assumed that the planets are composed of a material which is incompressible, both above and below the critical pressure, and in the second paper, by Lighthill, it is shown that the main conclusions of Ramsey's paper are valid also in the case of a compressible material. The problem is beset with many difficulties and, as Ramsey admits, in its more general form is mathematically cumbersome, but the assumption of incompressibility adds considerably to a simplified treatment of the subject. It is impossible in this article to do more than merely indicate some of the conclusions attained.

It is shown that the mass $M$ is normally an increasing function of the pressure $P$, that is, $d M / d P$ is normally positive. If the mass be $M_{0}$ when the critical pressure $p_{c}$ is attained for the phase transition at the boundary of the core, the derivative $d M / d P$ is discontinuous, and Lighthill shows that the derivative alters by a factor $(2 \lambda-3) / \lambda^{2}$, if the density jumps from $\rho_{0}$ to $\lambda \rho_{0}$ at the critical pressure, so that the derivative becomes negative if $\lambda$ exceeds $1 \cdot 5$. Hence the mass decreases below $M_{0}$, attains a maximum value $M_{1}$, and then increases again. As a consequence of this a planet can exist in three different equilibrium states if its mass lies between $M_{0}$ and $M_{1}$, which Ramsey denotes by $A, B$ and $C$. State $A$ has no core and states $B$ and $C$ have cores, that in state $C$ being the larger. While states $A$ and $C$ are stable, state $B$ is unstable, and a transition from $A$ to $C$ or from $C$ to $A$ is prevented by the energy barrier separating them. But if the mass is $M_{0}$ or $M_{1}$, a transition is possible; in the former case $A$ and $B$ coalesce, $C$ being the only stable state ; in the latter case $B$ and $C$ coalesce and $A$ is the only stable state. As the masses $M_{0}$ and $M_{1}$ depend on the thermal condition of the planet, and as the mean internal temperature of a planet may have changed by $10,000^{\circ}$ C. or more during its evolution, $M_{0}$ and $M_{2}$ could alter by about ten per cent. This possibility is important, because it is not essential that a planet should be in the critical mass range $M_{0}-M_{1}$ during its entire history. Moreover, this possible variation in temperature is not based on any theory of the origin of the solar system.

For the terrestrial planets $M_{0}$ is about $0.80 M_{E}$, where $M_{E}$ is the mass of the earth. While this is smaller than the mass of Venus by about 2 per cent, it is conceivable that $M_{0}$ could have changed by this amount during geological time, and hence Venus might have undergone a transition by this amount in this period; but Venus and the earth are the only planets to which this could have happened. A planet crossing the critical range, passing from on $\theta$ configuration to another, would release energy of the order $10^{37}$ ergs and could remove $0 \cdot 1-1 \cdot 0$ per cent of the mass from the surface, and blast-waves and vibrations of the planet as a whole would shatter the material, fragments flying off into space. It is 
tentatively suggested that this mechanism has been responsible for meteorites and possibly minor planets. It is admitted that the complexity of the problem prevents a complete quantitative treatment; and far from closing the question of the origin of these bodies, the present work should be regarded as re-opening this question.

${ }^{1}$ Ramsey, W. H., Nature, 168, 814 (1949), and 165, 217 (1950).

${ }^{2}$ Ramsey, W. H., Mor. Not. Roy. Astro. Soc., 108, 5 (1948).

\section{EXHIBITION SCHOLARS CENTENARY DINNER}

$\mathrm{R}$ ECENTLY in this Festival year in Great Britain a very special celebration was held: on the evening of May 1, exactly one hundred years after the date of the opening of the Great Exhibition in Hyde Park, about 130 men of science gathered together to dine and to do homage to the Prince Consort and other founders of that great achievement. All this company had one common bond between them : each had at some time in the early part of his career been awardod an 1851 Exhibition Scholarship which enabled him to continuo his postgraduate studies in scientific research.

As is well known, the 1851 Exhibition made a handsome profit, and this was first put to the purchase of a site in Kensington, which now houses the great museums of science and art, the Imperial College of Science and Technology and other educational institutions. Part of the site was developed and now brings in further substantial income. Ono of the projects which the Prince Consort had discussed was, however, not realized until thirty years after his death. In 1891 the Royal Commission for the Exhibition of 1851, under the inspiration of Lord Playfair, brought into being the first major scholarship scheme to aid young research students in Great Britain and in other parts of the Empire. Thus was started a fraternity of men of science, the total number of which now comes to nearly eight hundred, although unfortunately many of them are not now living. Out of these, more than three hundred went from the Dominions to Great Britain or other parts of Europe to complete their training. The members of the fraternity are now dispersed widely throughout the Commonwealth, the United States and other countries, where they play a leading part in academic and in applied science. Nearly eighty scholars have later in their career achieved the distinction of being elected to the Royal Society, and six have won Nobel Prizes.

The dinner on May 1 was held at University College, London, and the scholars had as their guests the members of the board of management of the Royal Commission and the members of the Science Scholarships Committee, together with a few others associated with the Exhibition trusteeship. The chair was taken by Lord Macmillan, who, after reading a message of goodwill from H.R.H. the Princess Royal, president of the Royal Commission, proposed a toast to the memory of the Prince Consort. In vivid style, Lord Macmillan described the difficulties that had to be overcome before the Great Exhibition could be started; he then recalled the words of Lord Playfair shortly after the Scholarship scheme was inaugurated, to the effect that if the scheme produced one Faraday a gift would have been presented to the country which would have been cheap at a million pounds. The career of Rutherford alone, a Scholar of 1895 , could be considered to have fulfilled Lord Playfeir's hopes, though there are a great many other 1851 Exhibition Scholars whose contributions to science are internationally recognized. A toast to the guests was proposed by Sir Irvine Masson (Scholar of 1910) and seconded by Dr. J. J. Sudborough (Scholar of 1891), one of the few survivors of the first group of appointments. These Scholars expressed the feelings of all those present in their appreciation of the valuable part 1851 Exhibition Scholarships had played in their own careers. The reply to their toast was made by Sir Henry Dale, and Sir Robert Robinson, who was present in the dual capacity of an 1851 Scholar and a member of the Science Scholarships Committee, expressed the company's thanks to the chairman.

The Scholars were particularly pleased to have with them that evening Sir Evelyn Shaw, who was secretary to the Commissioners during the period when most of the company had held their scholarships. He played a major part in the many happy reunions that went on that evening. Five hours later on the same day, a similar though smaller dinner was held in New York. Greetings were sent from the London dinner to Prof. H. S. Taylor for transmission to their New York colleagues. A further centenary dinner, for Canadian Scholars, was held in Montreal on June 2.

\section{BREEDING HABITS OF THE SWIFT}

$\mathrm{D}$ URING the years 1944-49, A. F. Cutcliffe and others made 250 visits to the tower of a church in Ilfracombe, Devon, to study the breeding habits of the swift (Apus apus); the visits were made at hours ranging from 6 a.m. to 12 midnight. An account of the observations appeared in a recent issue of British Birds (44, No. 2; February 1951).

The nests are situated above the bells at a height of $80 \mathrm{ft}$. from ground-level. Under the eaves of the tower on the east and west sides are a number of holes through the wall, some of which are occupied in early spring by nesting house-sparrows (Passer domesticus), which the swifts expel when they arrive, using these and any unoccupied apertures for breeding purposes. In this way the new arrivals find readymade nests to which they add other materials. One such nest contained three eggs laid by the sparrow owner before being evicted. The nests on the north and south sides of the church tower are made entirely by the swifts. Fourteen nesting sites have been used by breeding swifts during the period of observation, though not all have been occupied in any one year.

The first observations revealed that the nesting area was littered with skeletons of swifts, the casualties of past years, no fewer than thirty-eight complete skeletons, mostly of young birds, being counted. The nests and the surroundings contained many dark brown egg-capsules which were later identified as those of the two-winged fly, Cratorhina pallida.

The bases of the nests are constructed of hay and straw, the shallow cups being formed chiefly of small white feathers, identified as coming from gulls which abound in the district. Pieces of red and blue string 